\title{
2.5V Step-Down DCDCs: a Radiation-Hard Solution for Power Conversion
}

\author{
Giacomo Ripamonti ${ }^{1 a b}$, Stefano Michelis ${ }^{a}$, Federico Faccio ${ }^{a}$, Stefano Sagginic, \\ Mario Ursinoc, Georges Blanchot ${ }^{a}$, Stefano Caregariab, Adil Koukab ${ }^{b}$, Maher \\ Kayal $^{\mathrm{b}}$ \\ ${ }^{a} C E R N$ \\ ${ }^{b}$ École Polytechnique Fédérale de Lausanne \\ ${ }^{c}$ Università di Udine \\ E-mail: giacomo.ripamonti@cern.ch
}

\begin{abstract}
Radiation- and magnetic field tolerant DCDC converters that step down the voltage from a $2.5 \mathrm{~V}$ bus are needed for the High-Luminosity detectors. This work presents the developed prototypes, based on ASICs designed in a $130 \mathrm{~nm}$ CMOS technology. A buck converter (bPOL2V5) is close to production readiness, showing an $89.7 \%$ peak efficiency and tolerance to $200 \mathrm{Mrad}$ of Total Ionizing Dose (TID) and to a fluence of $10^{16} \mathrm{n} / \mathrm{cm}^{2}$. A lower-volume alternative to bPOL2V5 (called rPOL2V5) uses a resonant switched-capacitor architecture and shows comparable efficiency, while employing an eight times smaller inductor. The first prototype of rPOL2V5 has shown tolerance to more than $200 \mathrm{Mrad}$ of TID. For both converters, the ASIC and the PCB have been co-designed to guarantee high reliability.
\end{abstract}

Topical Workshop on Electronics for Particle Physics

2 - 6 September 2019

Santiago de Compostela, Spain

${ }^{1}$ Speaker

(C) Copyright owned by the author(s) under the terms of the Creative Commons 


\section{Introduction}

A lightweight and efficient power distribution system based on two stages of DC-to-DC conversion [1] was proposed for the front-end systems in the High-Luminosity Large Hadron Collider (HL-LHC) experiments that will use multiple power domains. A first-stage converter creates a $2.5 \mathrm{~V}$ domain from a $10 \mathrm{~V}$ line, powering the opto-electronic components. Second-stage converters further step down the voltage from $2.5 \mathrm{~V}$ to supply the front-end analog and digital circuits. All the converters are made of an ASIC and a few discrete components, among which an inductor. The environment in which such DCDC converters are placed requires them to be radiation and magnetic field tolerant. The large magnetic field (up to $4 \mathrm{~T}$ ) does not allow the use of inductors with ferro-magnetic core and their bulkier air-core counterparts must be employed. Any added material is detrimental for the physics performances of the experiments and minimizing the size of the inductor is therefore crucial.

This work focuses on second-stage converters, which must be able to step down the voltage from $2.0-2.5 \mathrm{~V}$ to $0.6-1.5 \mathrm{~V}$, providing a maximum output current of $3 \mathrm{~A}$. Such Point of Load (PoL) regulators must be tolerant to $150 \mathrm{Mrad}$ of Total Ionizing Dose (TID) and to a fluence of $7 \cdot 10^{15} \mathrm{n} / \mathrm{cm}^{2}$ for Displacement Damage. These are the radiation levels expected in the most exposed location where the converters will be installed (the outer trackers). Furthermore, Single Event Effects (SEEs) must not lead to destructive events and output power interruptions up to a Linear Energy Transfer of $40 \mathrm{MeV} \cdot \mathrm{cm}^{2} / \mathrm{mg}$. Two alternative converters using different architectures are in development for this application: the first (bPOL2V5) uses a buck architecture, while the second (rPOL2V5) is a resonant switched-capacitor DCDC equipped with a novel control circuit. This latter solution allows comparable efficiencies to the buck option, while using a significantly smaller inductor. Both alternatives are based on an ASIC designed in a $130 \mathrm{~nm}$ CMOS technology.

\section{2. bPOL2V5 (buck architecture)}

bPOL2V5 is a buck converter using a voltage-mode control. Its switching frequency is $4 \mathrm{MHz}$, which allows the use a $100 \mathrm{nH}$ inductor. During the switching operation, large $d I / d t$ occur in the bonding and PCB parasitic inductances along the input current path, causing voltage spikes that exceed $V_{\text {in }}$. Such overvoltages can affect the reliability of the used MOSFET devices. A flipchip assembly has been adopted to reduce the bonding inductance. In addition, an in-depth study has led to the design of an optimized PCB featuring a minimal input inductance. Finally, the turnon and turn-off times of the power switches have been increased to reduce the input $d I / d t$. Early prototypes of bPOL2V5 used 2.5 V-rated MOSFETs [2], and even the countermeasures presented above could not avoid that overvoltages exceeding the device rating were present during the converter operation. Therefore, 3.3 V-rated transistors have been chosen for the last prototypes and will be used in production.

In the last version of bPOL2V5, the bandgap voltage reference that is used to determine the value of $V_{\text {out }}$ can be trimmed, allowing an accuracy of $1 \%$ on the output voltage. Furthermore, the converter is protected from an excessive load current $\left(I_{\text {ou }}>3.8 \mathrm{~A}\right)$ thanks to an on-chip overcurrent protection. The ASIC includes a temperature sensor and an overtemperature protection, which prevents the converter from working above $100^{\circ} \mathrm{C}$. Finally, bPOL2V 5 features a novel pulse width 

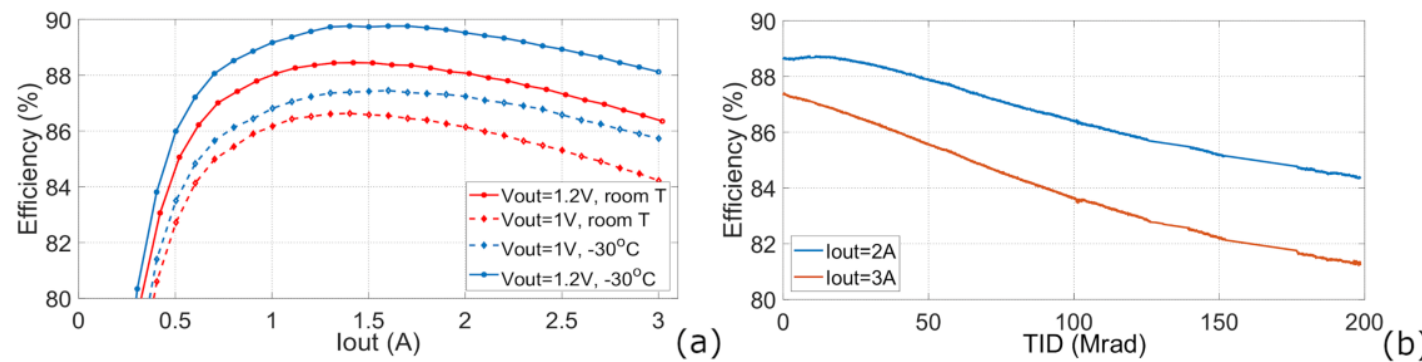

Figure 1. (a) Measured efficiency of the last prototype of bPOL2V5, with $V_{i n}=2.5 \mathrm{~V}$. (b) Evolution of the efficiency of the last prototype of bPOL2V5 during an X-ray irradiation using a dose rate of $2 \mathrm{Mrad} / \mathrm{h}$ at $-30^{\circ} \mathrm{C}$, with $V_{\text {in }}=2.5 \mathrm{~V}$ and $V_{\text {out }}=1.2 \mathrm{~V}$.

modulator that guarantees improved dynamic performances [3].

\subsection{Measurement results on bPOL2V5}

The last prototype of bPOL2V5 exhibits output voltage variations of less than $1 \mathrm{mV}$ in the application range of the line voltage and load current. The prototype uses a $100 \mathrm{nH}$ inductor and can reach a peak efficiency of $89.7 \%$ at $-30^{\circ} \mathrm{C}$ for the $2.5 \mathrm{~V}$-to- $1.2 \mathrm{~V}$ conversion (see Fig. 1a), the efficiency decreasing for larger $V_{\text {in }} / V_{\text {out }}$ ratios. Stress tests at an input voltage of $3 \mathrm{~V}$ have been carried out on 32 samples for $1000 \mathrm{~h}$ ( 16 samples were at full load, $I_{\text {out }}=3 \mathrm{~A}$, while the remaining samples had $I_{\text {out }}=1.2 \mathrm{~A}$ ). No damage was observed in any of the stressed converters.

TID irradiation was performed on six samples in different conditions using an X-ray system. Fig. $1 \mathrm{~b}$ shows an example result for a converter exposed at $-30^{\circ} \mathrm{C}$ up to a TID of $200 \mathrm{Mrad}\left(\mathrm{SiO}_{2}\right)$. The sample was fully functional during the whole test and consequent annealing. $V_{\text {out }}$ increased by less than $20 \mathrm{mV}$ during the irradiation, while the efficiency for the $2.5 \mathrm{~V}$-to- $1.2 \mathrm{~V}$ was degraded by $6 \%$ at $I_{\text {out }}=3 \mathrm{~A}$ and by $4 \%$ at $I_{\text {out }}=2 \mathrm{~A}$ (see Fig. $1 \mathrm{~b}$ ). It has been found that such efficiency degradation is lower for a lower dose rate. Since the dose rate used for these tests ( $2 \mathrm{Mrad} / \mathrm{h}$ ) is significantly larger than the one expected in the HL-LHC detectors, a reduced efficiency degradation is expected in the application. In order to evaluate the effects of Displacement Damage, a previous prototype using 3.3 V-rated devices has also been irradiated both with protons at the IRRAD facility at CERN, up to a fluence of $6.6 \cdot 10^{15} \mathrm{p} / \mathrm{cm}^{2}$, and with neutrons at the TRIGA reactor in Ljubljana, up to $10^{16} \mathrm{n} / \mathrm{cm}^{2}$. Such irradiations have revealed no significant degradation in the performances.

\section{3. rPOL2V5 (resonant switched-capacitor architecture)}

Switched-capacitor (SC) DCDC converters do not use any magnetic components, and they have therefore a larger power density compared to their inductor-based counterparts. Nevertheless, their efficiency is limited by the charge redistribution losses that are inherent to their operation. Resonant switched-capacitor (ReSC) converters result from the addition of a small inductor to a SC converter, which allows overcoming the charge redistribution losses, while keeping increased power density compared to inductor-based solutions [4]. rPOL2 V5 is a ReSC converter that can reach efficiencies comparable to bPOL2V5, while having a significantly lower volume (it can use a $12 \mathrm{nH}$ inductor). The schematic of its power stage is shown in Fig. 2a. Two out of the four power switches are always on and share the voltage stress, allowing the use of the more radiation-hard $2.5 \mathrm{~V}$-rated devices. 


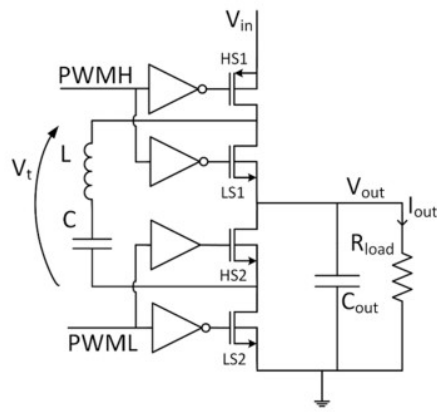

(a)
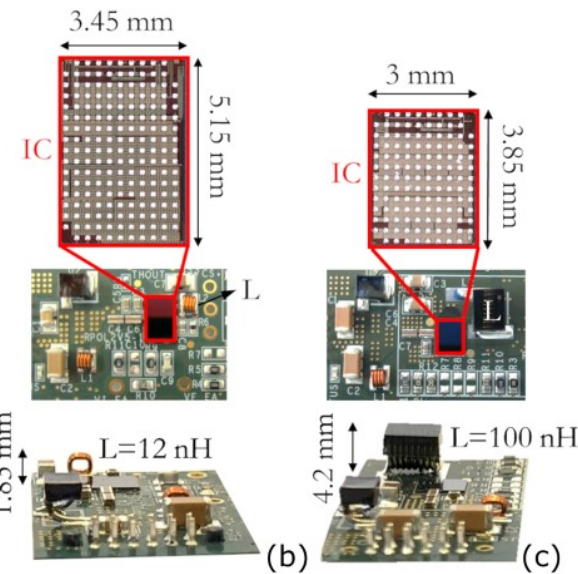

Figure 2. (a) Schematic of the power stage of rPOL2V5. (b) Pictures of the rPOL2V5 ASIC and test module, and (c) of the bPOL2V5 ASIC and test module.

rPOL2V5 achieves output voltage regulation through the modulation of the LC tank excitation voltage $V_{t}$ : according to the required load current, the control selects the amplitude and frequency of $V_{t}$. This allows the tank to generate the proper current, while keeping a constant $V_{\text {out }}$. The novel controller developed for rPOL2V5 implements different operation modes that optimize the efficiency for the full range of load currents [5]. In particular, the mode used at heavy loads is inherently inefficient for light loads, where two alternative modes can be used: on one hand, the light load operation provides the best efficiency and the smallest output ripple, but it is not functional for $V_{\text {out }}>V_{\text {in }} / 2$. The burst mode works instead for all conversion ratios, but it exhibits a lower efficiency and an increased output ripple.

A first prototype of rPOL2V5 has been developed, adopting a flip-chip assembly and an optimized PCB layout to minimize the input parasitic inductance and the related overvoltages.
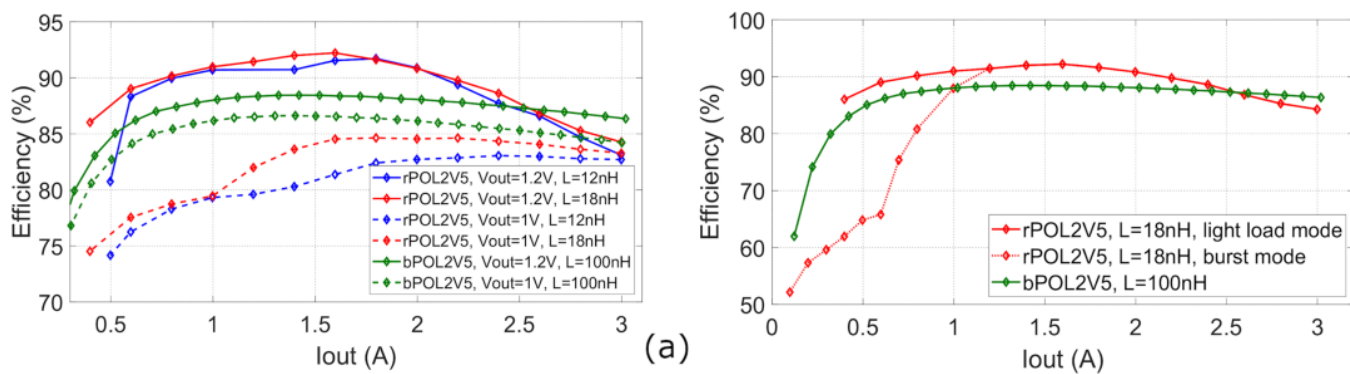

Figure 3. (a) Measured efficiencies of rPOL2V5 vs. bPOL2V5, at room temperature and for $V_{\text {in }}=2.5 \mathrm{~V}$. (b) Efficiency comparison between light load mode and burst mode, at room temperature, for $V_{\text {in }}=2.5 \mathrm{~V}$ and $V_{\text {out }}=1.2 \mathrm{~V}$.
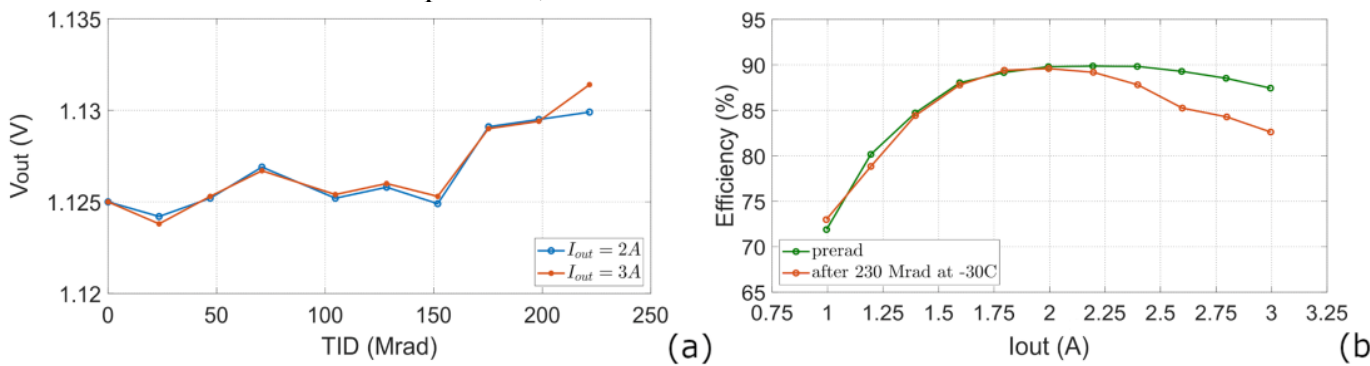

Figure 4. Evolution (a) of the output voltage of rPOL2V during an X-ray irradiation at $-30^{\circ} \mathrm{C}$, with $V_{\text {in }}=2.5 \mathrm{~V}$. (b) Comparison between the efficiency before and after the irradiation, at room temperature $\left(V_{\text {in }}=2.5 \mathrm{~V}, V_{\text {out }}=1.14 \mathrm{~V}\right)$. 
Fig. 2b-c respectively show the test modules of rPOL2V5 and bPOL2V5. The $12 \mathrm{nH}$ inductor used for rPOL2V5 has a significantly lower volume than the $100 \mathrm{nH}$ of the buck version, and it is particularly interesting for applications that need a low-profile DCDC converter.

\subsection{Measurement results on $\mathrm{rPOL2V5}$}

The measured efficiency of the first prototype of rPOL2V5 is presented in Fig. 3a: for the $2.5 \mathrm{~V}$-to-1.2 V conversion, rPOL2V5 achieves a peak efficiency of $92.2 \%$, which is larger than that of bPOL2V5. This ReSC converter exhibits its best performances when $V_{\text {out }}$ is slightly lower than $V_{i n} / 2$. Fig. $3 \mathrm{~b}$ presents the comparison between the efficiencies at light loads obtained with the light load mode and the burst mode: although the latter has a lower efficiency, measurements have shown that its operation is more reliable than that of the former. Startup issues have been found for very light loads, while also the operation at heavy loads for $V_{\text {out }}>V_{\text {in }} / 2$ must be improved. The proper countermeasures to avoid these two issues in a future prototype have been identified.

An irradiation with X-rays up to $230 \mathrm{Mrad}\left(\mathrm{SiO}_{2}\right)$ has shown that rPOL2V5 meets the target for TID tolerance: Fig. 4 a shows that the output voltage is stable during the irradiation, while Fig. $4 \mathrm{~b}$ highlights that the radiation-induced efficiency degradation is larger at heavy loads.

\section{Outlook and conclusion}

Two 2.5 V step-down DCDC converters based on radiation-tolerant ASICs are in development for the HL-LHC experiments.

bPOL2V5 is a buck converter whose last prototype complies with the electrical specifications, exhibiting a peak efficiency of $89.7 \%$. In addition, it tolerates $200 \mathrm{Mrad}$ of TID and a fluence of $10^{16} \mathrm{n} / \mathrm{cm}^{2}$. It must be verified that it is tolerant to SEEs before launching the final production.

rPOL2V5 is a resonant switched-capacitor converter whose first prototype has shown an increased peak efficiency compared to bPOL2V5 (92.2\%), while having a reduced volume thanks to its eight times smaller inductor. It has been demonstrated that rPOL2V5 is tolerant to $230 \mathrm{Mrad}$ of TID. To finalize its radiation characterization, Displacement Damage and SEE tests are necessary. By introducing a few improvements, a second prototype of rPOL2V5 could be close to production readiness.

\section{References}

[1] F. Faccio, S. Michelis, Custom DC-DC converters for distributing power in SLHC trackers, in proceedings of TWEPP, Naxos, Greece, 2008.

[2] G. Ripamonti et al., A 2.5V Step-Down DC-DC converter for Two-Stages Power Distribution Systems, PoS (TWEPP-17) 059.

[3] G. Ripamonti et al., A Dual-Edge Pulsewidth Modulator for Fast Dynamic Response DC-DC Converters, IEEE Transactions on Power Electronics, vol. 34, no. 1, pp. 28-32, Jan. 2019.

[4] K. Kesarwani, R. Sangwan and J. T. Stauth, Resonant-Switched Capacitor Converters for ChipScale Power Delivery: Design and Implementation, in IEEE Transactions on Power Electronics, vol. 30, no. 12, pp. 6966-6977, Dec. 2015.

[5] G. Ripamonti et al., An Integrated Regulated Resonant Switched-Capacitor DC-DC Converter for PoL Applications, in proceedings of IEEE APEC, Anaheim, CA, USA, pp. 207-211, March 2019. 